\title{
El tamaño de los individuos y el microambiente afectan el daño por fuego y la supervivencia en árboles del Chaco Serrano
}

\author{
Julieta Alinari ${ }^{\prime}$; Ana M. Cingolani ${ }^{2} \bowtie$; Axel R. von Müller ${ }^{3} \&$ Marcelo Cabido $^{2}$ \\ ${ }^{1}$ Centro de Investigación y Extensión Forestal Andino Patagónico. Esquel, Chubut, Argentina. ${ }^{2}$ Instituto Multidisciplinario \\ de Biología Vegetal (CONICET-Universidad Nacional de Córdoba). Córdoba, Argentina. ${ }^{3}$ Instituto Nacional de Tecnología \\ Agropecuaria, Estación Experimental Agroforestal Esquel. Chubut, Argentina.
}

\begin{abstract}
Resumen. Los incendios afectan de distinta manera a ejemplares de una misma especie leñosa según el tamaño del individuo y las condiciones del microambiente, entre otros factores. Esto tiene implicancias en la estructuración de los paisajes forestales, ya que la velocidad a la que se recupera el bosque depende, en parte, de la severidad con la cual el fuego afecta a los individuos leñosos. Nos propusimos analizar cómo el tamaño y el microambiente afectan el nivel de daño por fuego y la supervivencia en dos especies leñosas características del bosque chaqueño serrano, en un gradiente altitudinal en las Sierras de Córdoba (Argentina). Estudiamos dos incendios que ocurrieron entre julio y agosto de 2007. Seleccionamos 163 espinillos (Vachellia caven) y 48 molles (Lithraea molleoides) localizados entre 800 y $1700 \mathrm{~m}$ s. n. m. Para cada individuo estimamos la altura y el volumen pre-fuego en base al leño remanente, y características del microambiente como cobertura vegetal, proporción de roca bajo la copa, pendiente, orientación de la ladera e insolación por intercepción. Tres meses después estimamos el daño por fuego y tres años más tarde evaluamos la supervivencia. Los espinillos con más vegetación en su ambiente circundante sufrieron más daño por el fuego, y este efecto fue más pronunciado a menores altitudes. Además, los ejemplares de las laderas sur con mucha pendiente sufrieron más daño que los de las laderas norte, y los individuos más pequeños resultaron más dañados que los grandes. Los molles más altos sufrieron menos daño por fuego que los más bajos. El $94 \%$ de los espinillos y el $92 \%$ de los molles sobrevivieron al incendio, $y$, en el caso del espinillo, la probabilidad de morir fue mayor para los ejemplares más pequeños. Concluimos que el sistema se vuelve menos susceptible al fuego a medida que avanza la sucesión, al aumentar el tamaño de los individuos y disminuir la cobertura herbácea.
\end{abstract}

[Palabras clave: altitud, altura del árbol, bosque serrano, cobertura vegetal, espinillo, incendios, molle, pendiente, rocosidad, Sierras de Córdoba]

\begin{abstract}
Aвstract. Tree size and microenvironment affect fire damage and survival in Chaco Serrano. Wildfires differentially affect specimens of the same woody species, depending, among other factors, on tree size and microenvironment. This has implications for the structuring of forest landscapes, as forest recovery depends, in part, on the severity of fire on woody specimens. We aimed at analyzing how microenvironment and tree size affect fire damage and survival in two woody species common in the Chaco Serrano forest, along an altitudinal gradient in Córdoba Mountains (Argentina). We studied two wildfires that occurred in July and August 2007. We selected 163 espinillos (Vachellia caven) and 48 molles (Lithraea molleoides) located between 800 and $1700 \mathrm{~m}$ a. s. 1 . For each individual, we estimated pre-fire height and volume on the basis of the remaining woody tissues, and microenvironmental characteristics as plant cover, rock proportion under the crown, slope, aspect and insolation by interception. After three months we estimated the fire damage for each individual and three years later we evaluated survival. The espinillos with higher plant cover in their surroundings were more damaged, and this effect was more pronounced at lower altitudes. Additionally, individuals were more damaged in steep southern slopes than in northern slopes, while smaller individuals were more damaged than larger ones. Taller molles experienced less fire damage than shorter molles. The $94 \%$ of the espinillos and $92 \%$ of the molles survived the wildfire, with higher death probability for smaller individuals in the case of espinillo. We concluded the system becomes less susceptible to fire as succession proceeds, mainly because of the increasing size of woody individuals and the reduction of herbaceous cover.
\end{abstract}

[Keywords: altitude, tree height, mountain forest, plant cover, espinillo, wildfires, molle, slope, rockiness, Córdoba Mountains] 


\section{INTRODUCCIÓN}

En muchos ecosistemas, el fuego es uno de los disturbios que más influyen sobre la estructura de la vegetación (Veblen et al. 1999; Oliveira and Pillar 2004; Bond et al. 2005). En aquellos bosques cuyas plantas leñosas suelen rebrotar después de los incendios, el fuego no produce cambios sustanciales en la composición de especies, pero sí en la fisonomía, debido a la drástica reducción de la biomasa arbórea que favorece la proliferación de hierbas y de arbustos (Türkmen and Düzenli 2005; Giorgis et al. 2017). Sin embargo, un mismo incendio puede afectar de distinta manera a ejemplares de una misma especie leñosa, dependiendo, entre otras cosas, del tamaño de los individuos y de las condiciones del microambiente (Marañón et al. 2004; Alinari et al. 2015; Landesmann et al. 2015). De este modo, los tejidos de algunos árboles resultan muy dañados, mientras que los de otros pueden sufrir menos daño. Los individuos más dañados perderán más biomasa aérea y tendrán menos probabilidad de sobrevivir al fuego o, si sobreviven, tardarán más tiempo en recuperar su biomasa en comparación con los individuos menos dañados (Cable 1965; Renison et al. 2002; Ordoñez et al. 2005; Alinari et al. 2015). Por estas razones, el tamaño de los árboles y las condiciones del microambiente, al influir sobre el daño de los tejidos, pueden afectar la velocidad a la cual el bosque recupera su estructura anterior al fuego. En sistemas con fuegos recurrentes, la velocidad de recuperación determina el tipo de cobertura vegetal que predomina en el paisaje (Kitzberger et al. 2016; Tepley et al. 2018). Por este motivo, entender cómo el microambiente y el tamaño de los árboles influyen sobre el daño que éstos experimentan debido al fuego contribuye a conocer los factores estructuradores de los paisajes. Por ejemplo, en distintos ecosistemas de Sudamérica se observó que proliferaban especies leñosas por sobre las herbáceas en lugares donde predominan microambientes protegidos del fuego y la ganadería; esto se debió, en parte, a una menor mortalidad o una mayor velocidad de recuperación post-disturbio de dichas especies en esos ambientes (Renison et al. 2006; Carlucci et al. 2011; Landesmann et al. 2015; Alinari et al. 2015).

Numerosos estudios destacan la importancia del tamaño de los individuos. En el caso de fuegos de superficie, si el microambiente es uniforme, los árboles más altos o más voluminosos pueden escapar parcialmente del fuego, mientras que árboles más pequeños pueden experimentar mayor daño dado que las llamas los alcanzan fácilmente (Cable 1965; Ordoñez et al. 2005; Fernandes et al. 2008; Brando et al. 2012). Además, el tamaño del individuo puede afectar la vegetación que lo rodea. Las copas de los árboles más altos o frondosos reducen la entrada de luz, lo que limita la acumulación de biomasa y la continuidad vertical del material combustible; esto evita que las llamas alcancen la copa (Kitzberger et al. 2016; Blackhall et al. 2015a,b). Por estas razones, una vez que se pierde la estructura boscosa, en muchos casos el sistema se vuelve más inflamable y aumenta la recurrencia de los fuegos, lo cual impide el desarrollo de los árboles. Así se crea una retroalimentación positiva entre el fuego y la vegetación, y el sistema se mantiene en un estado permanente de matorral o pastizal (Blanco et al. 2014; Landesmann et al. 2015; Tepley et al. 2018).

También el microambiente juega un papel fundamental. Por un lado, la cantidad y el tipo de vegetación que rodea al árbol pueden influir en la propagación de los fuegos de superficie y su intensidad y, por lo tanto, en el daño que experimenta el ejemplar (Keeley 2009). Como se mencionó, la vegetación circundante puede estar influenciada por las características de los mismos árboles, pero también es afectada por otros factores. Por ejemplo, si el ejemplar está rodeado por rocas que impiden el desarrollo de la vegetación, sus tejidos aéreos pueden escapar total o parcialmente al daño, y esto aumenta la probabilidad de supervivencia y facilita la recuperación posterior (Renison et al. 2002; Carlucci et al. 2011; Alinari et al. 2015; Landesmann et al. 2015). Por el contrario, la biomasa aérea de árboles similares puede quemarse por completo si éstos están rodeados por gramíneas que constituyen combustibles finos de alta inflamabilidad (Renison et al. 2002; Jaureguiberry et al. 2011). Asimismo, la topografía influye sobre la humedad del suelo y, por lo tanto, sobre la humedad del material combustible (Parker 1982; Flatley et al. 2011). En las laderas con mayor pendiente, el agua infiltra menos y escurre pendiente abajo; cuando infiltra, se pierde del suelo más rápidamente por transporte subsuperficial. Además, si estas laderas reciben una insolación mayor por estar orientadas al norte (en el hemisferio sur) o en posiciones topográficas altas, la desecación del suelo y de la vegetación es aun mayor. Contrariamente, 
en lugares con menor pendiente el agua infiltra más en el suelo y, además, se pierde menos por flujo sub-superficial. Si los sitios reciben menos insolación, la pérdida de humedad es aun menor. Además, los sitios en posiciones topográficas bajas reciben un aporte de agua adicional resultado del escurrimiento y el transporte sub-superficial desde posiciones más altas (Parker 1982). De este modo, en los micrositios con suelos secos que favorecen la desecación del material combustible la inflamabilidad es mayor, y por lo tanto los fuegos suelen ser más intensos que en los micrositios con suelos más húmedos (Blackhall et al. 2012, 2015a; Bianchi and Defossé 2015; Bianchi et al. 2014).

En sistemas montañosos existe una variabilidad marcada en el tamaño de los individuos de especies leñosas y en las características del microambiente que los rodea (Veblen et al. 1999; Kupferschmid and Bugmann 2005). Esta variabilidad puede traer aparejada una alta heterogeneidad en la manera en que los individuos de una misma especie son afectados por el fuego (Renison et al. 2002; Landesmann et al. 2015). A su vez, en sistemas montañosos, la altitud puede influenciar la humedad del suelo, la rocosidad y la vegetación (Alinari et al. 2015; Tecco et al. 2015). Por lo tanto, es posible que el efecto del tamaño del árbol y del microambiente sobre el daño por fuego sea diferente a distintas altitudes. En síntesis, los antecedentes indican que los árboles se ven dañados por el fuego en distinto grado, dependiendo de su tamaño y de las características microambientales que los rodean, y esto, a su vez, puede estar influenciado por la altitud. Todo ello puede afectar la supervivencia y posterior recuperación de los ejemplares leñosos y, por lo tanto, tener implicancias importantes para la distribución actual de los bosques. En este trabajo nos propusimos estudiar el efecto del tamaño del árbol y del microambiente que lo rodea sobre el daño experimentado por el fuego y la supervivencia post-fuego para dos especies características del bosque chaqueño serrano en la Argentina, a lo largo de un gradiente altitudinal. Esperamos que los árboles más grandes, rodeados por menos vegetación y más roca, y ubicados en situaciones topográficas que favorecen la mayor humedad del suelo (menos pendiente y menos insolación) experimenten un menor daño que árboles en situaciones opuestas. A su vez, esperamos que los árboles que sufren menos daño tengan una mayor probabilidad de sobrevivir que aquellos que sufren más daño.

\section{Materiales y Métodos}

\section{Área y especies estudiadas}

El estudio se llevó a cabo en la ladera occidental del cerro Champaquí, en las Sierras Grandes de Córdoba (Argentina), a lo largo de un gradiente altitudinal entre 800 y 1700 m s. n. m. (punto central del área de estudio: $32^{\circ} 02^{\prime} 39^{\prime \prime} \mathrm{S}$ - 64 $\left.54^{\circ} 37^{\prime \prime} \mathrm{O}\right)$. En las Sierras de Córdoba, desde los 500 hasta los 1700 m s. n. m. la vegetación incluye un mosaico de bosques, matorrales y pastizales dominados por especies características del piso altitudinal del bosque chaqueño serrano (Kurtz 1904; Giorgis et al. 2017). Los bosques alcanzan alturas de 8 a $12 \mathrm{~m}$, mientras que los matorrales son sucesionales y suelen tener entre 3 y $5 \mathrm{~m}$ de altura (Giorgis et al. 2017). Si bien en las zonas más altas dentro de este rango altitudinal la cobertura arbórea es baja, se pueden encontrar pequeños parches de bosques, matorrales e individuos aislados de las especies leñosas características, entre ellas el molle de beber (Lithraea molleoides (Vell.) Engl.) y el espinillo (Vachellia caven (Molina) Seigler and Ebinger) (Giorgis et al. 2013, 2017). Por encima de los 1700 m s. n. m., la vegetación se relaciona más con las floras andina y patagónica que con la chaqueña, y el árbol dominante es el tabaquillo (Polylepis australis Bitter) (Cabido et al. 1998; Martínez et al. 2017; Cabido et al. 2018). Esta especie puede bajar formando bosquecitos pequeños en laderas sur hasta los $1200 \mathrm{~m} \mathrm{~s}$. n. m., en algunos casos compartiendo la dominancia con Maytenus boaria Molina y especies del Chaco Serrano (Marcora et al. 2008).

El clima de las sierras está gobernado por el gradiente de altitud. A $527 \mathrm{~m} \mathrm{s.} \mathrm{n.} \mathrm{m.,} \mathrm{en} \mathrm{la}$ Estación Experimental del INTA Villa Dolores (21.5 km al oeste del área de estudio), la temperatura media anual es $18.9^{\circ} \mathrm{C}$, mientras que a $1800 \mathrm{~m} \mathrm{s.} \mathrm{n.} \mathrm{m.,} \mathrm{en} \mathrm{la} \mathrm{ladera} \mathrm{oriental} \mathrm{del}$ cerro Champaquí (10 km del área de estudio), la temperatura media es $11.2^{\circ} \mathrm{C}$. A $2700 \mathrm{~m} \mathrm{~s}$. $n$. $\mathrm{m}$., casi en la cumbre del mismo cerro, es de 7.4 ${ }^{\circ} \mathrm{C}$ (INTA, comunicación personal) (Marcora et al. 2008). Las lluvias están concentradas en los meses más cálidos (Colladon and Pazos 2014). En Villa Dolores, el promedio histórico es de $682 \mathrm{~mm} /$ año (período 1961-2006) (INTA 2007), pero a medida que se asciende en altitud las lluvias pueden alcanzar los $900 \mathrm{~mm} /$ año 
y la humedad del suelo es mayor (Giorgis et al. 2017; Tecco et al. 2015).

En su gran mayoría, los incendios en las Sierras de Córdoba son antropogénicos y ocurren todos los años a lo largo de todo el gradiente altitudinal. En un estudio que abarcó alrededor de $24000 \mathrm{~km}^{2}$, incluyendo cuatro sistemas serranos, Argañaraz et al. $(2015 a, b)$ encontraron que en un período de 13 años se quemó un 19\% del área, en algunos casos más de una vez. En áreas poco rocosas donde predominan pastizales y matorrales sucesionales, la recurrencia promedio del fuego es de 30 a 40 años (calculada sobre la base de los trabajos citados y J. Argañaraz comunicación personal). Sin embargo, localmente, en algunos sectores próximos a asentamientos humanos (e.g., en los alrededores de basurales), la frecuencia de incendios puede llegar a ser mucho más alta, de hasta un incendio cada tres o cuatro años (Argañaraz et al. 2015a,b). Dado que las especies leñosas del bosque serrano son de crecimiento lento (alrededor de $10 \mathrm{~cm}$ por año) (Capó et al. 2016), los fuegos suelen afectarlas antes de que los individuos logren alcanzar un gran tamaño. Si bien la gran mayoría de los árboles rebrota después de perder su biomasa, el daño producido disminuye drásticamente su tamaño (Torres et al. 2014). Por esta razón, la presencia deárboles grandes y, eventualmente, de bosques maduros, depende en parte de que haya microambientes donde los ejemplares resulten menos dañados y puedan escapar, al menos parcialmente, del fuego.

En la ladera occidental del cerro Champaquí ocurrieron dos incendios de superficie entre los meses de julio y agosto de 2007. Uno de ellos tuvo lugar cerca del poblado de San Javier (estancia La Constancia) y el otro en las proximidades de la localidad de La Población (estancia El Carrizal). El fuego de la estancia El Carrizal se extendió desde los $800 \mathrm{~m} \mathrm{s.} \mathrm{n.}$ $\mathrm{m}$. hasta el filo de las sierras, a $2700 \mathrm{~m} \mathrm{s.} \mathrm{n.} \mathrm{m.}$ (552 ha), mientras que de La Constancia se extendió desde los 1100 hasta más de $1700 \mathrm{~m}$ s. n. m. (353 ha). Ambos incendios se iniciaron en la zona más baja y se propagaron hacia arriba. Para poder contar con el suficiente número de individuos para realizar el estudio consideramos ambas zonas en conjunto, ya que los dos sitios son similares en su topografía, y la vegetación en ambos casos consistió en matorrales, pastizales y algunos pequeños parches de bosques característicos del Chaco Serrano (Giorgis et al. 2017). Entre ambos poblados hay una distancia no mayor a seis kilómetros en línea recta, por lo que consideramos el gradiente como un continuo. En toda el área de estudio hay presencia de ganado doméstico, principalmente bovino (Alinari 2017).

Seleccionamos individuos de dos especies que son dominantes en las comunidades de bosques y matorrales del Chaco Serrano: molle de beber (Lithraea molleoides) y espinillo (Vachellia caven). El molle es dominante en los bosques cerrados y en los abiertos, y también está presente en forma de individuos medianos y pequeños en los matorrales y pastizales sucesionales. El espinillo es un árbol pequeño o arbusto dominante en los matorrales sucesionales, pero también está presente en pastizales y bosques abiertos. Esta especie está prácticamente ausente en los bosques cerrados con árboles de gran porte, ya que no tolera la sombra (Torres et al. 2015; Giorgis et al. 2017; Cabido et al. 2018).

\section{Daño y supervivencia}

Dos a tres meses después del incendio (octubre 2007), antes del rebrote de la vegetación, seleccionamos individuos de ambas especies dentro del área quemada. Para estratificar la selección de individuos dividimos el gradiente altitudinal $(800-1700 \mathrm{~m}$ s. n. m.) en nueve franjas de $\sim 100 \mathrm{~m}$. Dentro de cada franja marcamos entre 11 y 21 espinillos. En el caso del molle, sólo pudimos seleccionar individuos en las primeras cuatro franjas, entre los 800 y 1200 m s. n. m. (11 a 13 individuos por franja). Si bien por encima de esta altitud había ejemplares de esta especie, la cantidad no era suficiente como para incluirlos en el estudio. En total, seleccionamos 163 espinillos y 48 molles mayores a $80 \mathrm{~cm}$ de altura. Para cada árbol estimamos el nivel de daño (\%) identificando visualmente la proporción de leño remanente carbonizado (Renison et al. 2002). Por ejemplo, si la cuarta parte del leño remanente mostraba una superficie carbonizada, estimamos el daño como $25 \%$. Luego de los fuegos de superficie, el leño de la mayoría de los árboles permanece en pie, carbonizado o no, incluyendo ramas bastante finas. En general, las ramas no carbonizadas mantienen el follaje verde, mientras que las ramas carbonizadas presentan el follaje muerto, o no lo presentan. En muy pocos casos de individuos pequeños se detecta la pérdida total del leño y se puede observar sólo la base carbonizada. Estos pocos individuos fueron 
descartados al hacer la selección porque la ausencia de leño en pie impedía estimar el tamaño pre-fuego.

En cada franja altitudinal procuramos seleccionar individuos dentro de todo el rango posible de niveles de daño (desde $<25 \%$ hasta $>75 \%$ ), lo cual fue posible para espinillo, pero no para molle. En el caso del molle, una gran parte de los árboles se quemaron por completo, y sólo una fracción escapó parcialmente al fuego. Por este motivo, los individuos seleccionados se clasificaron en dos categorías: con daño total (38 árboles, todos dañados en un $100 \%$ ) y con daño parcial (10 árboles, que variaron entre 5 y 80\% de daño). $\mathrm{Si}$ bien intentamos que hubiera individuos de ambas categorías en las cuatro franjas altitudinales, en la parte más alta encontramos muy pocos molles con daño parcial. Tres años después del incendio (invierno de 2010) estimamos la supervivencia de los individuos. Para ello visitamos cada árbol y registramos si presentaba ramas y hojas vivas.

\section{Tamaño y microambiente}

Al momento de hacer la selección (octubre 2007) medimos la altura pre-fuego desde el suelo hasta la rama más alta $(\mathrm{cm})$ de todos los ejemplares de ambas especies considerando el leño remanente tanto vivo como muerto (Renison et al. 2002; Gurvich et al. 2005). También medimos el ancho mayor de la copa (la distancia horizontal entre los dos puntos más alejados de la copa) y el ancho perpendicular a este último $(\mathrm{cm})$. Con estos datos calculamos un "índice de volumen" pre-fuego para complementar la información brindada por la altura, como la raíz cúbica del producto entre las tres dimensiones del árbol:

Índice de volumen $=($ altura $\mathrm{x}$ ancho mayor $\mathrm{x}$ ancho perpendicular)

En el invierno de 2008, un año después del fuego, evaluamos las condiciones microambientales en las que crecían los individuos. Estimamos visualmente la proporción (\%) de suelo expuesto, pedregullo y roca, y la cobertura (\%) de la vegetación a dos escalas: bajo la copa y en el ambiente circundante (área circular de $5 \mathrm{~m}$ de radio centrada en el tronco del ejemplar). Consideramos como suelo expuesto al sustrato conformado por partículas menores a $2 \mathrm{~mm}$ (USDA 2017), pedregullo al sustrato conformado por partículas entre $2 \mathrm{~mm}$ y $200 \mathrm{~mm}$, y roca al sustrato conformado por partículas mayores a $200 \mathrm{~mm}$ (estimación táctil y visual). Si bien es posible que estos datos no reflejen con exactitud las coberturas anteriores al incendio, consideramos que son indicadores confiables en términos relativos. Es decir, es probable que los árboles rodeados por mayor proporción de roca en la fecha de medición también hayan estado rodeados por una mayor proporción de roca antes del incendio, y dado que la vegetación herbácea y leñosa dominante tiende a rebrotar, asumimos que una mayor cobertura vegetal posterior (un año después) al fuego indica que también había una mayor cobertura vegetal antes del fuego.

Además, medimos la pendiente (\%) y la orientación de la ladera $\left(^{\circ}\right)$ con clinómetro y brújula, respectivamente. Calculamos la orientación relativa al norte a través del coseno de la orientación. Los valores negativos indican orientaciones sur, mientras que los valores cercanos a cero indican orientaciones este $u$ oeste (ningún micrositio fue completamente llano), y los valores positivos, orientaciones norte. La orientación relativa al norte combinada con la pendiente se relaciona con el ángulo de incidencia de los rayos solares durante gran parte del año y, por lo tanto, con la insolación que recibe un sitio si no hay intercepción de accidentes topográficos (Cingolani et al. 2014). Además, estimamos la insolación por intercepción utilizando un clinómetro $\left({ }^{\circ}\right)$. Ésta se estimó como la diferencia entre el plano horizontal $\left(180^{\circ}\right)$ y el ángulo que impide la incidencia del sol en el sitio debido a accidentes topográficos o rocas tanto hacia el este como al oeste (Alinari et al. 2015). Esta última variable es también un indicador de la posición topográfica, ya que tiene mayores valores en los sitios más altos en el paisaje (cumbres de lomas) y valores bajos en las posiciones inferiores en el paisaje (valles).

\section{Análisis de datos}

Analizamos el efecto del microambiente y el tamaño del individuo, así como la interacción de estas variables con la altitud, sobre el daño sufrido por ambas especies; para ello usamos modelos lineares generalizados con distribución beta (espinillo) o binomial (molle) y enlace logit. En el caso del espinillo, la variable dependiente fue el nivel de daño, expresado como proporción, y en el caso del molle, la variable dependiente fue el nivel de daño, categorizado como 0 ó 1 (con daño parcial o total respectivamente). Como variables independientes consideramos la 
altura del árbol $(\mathrm{cm})$ o el índice de volumen (cm) anteriores al fuego, junto con las variables microambientales. Estas últimas consistieron en la proporción de roca (\%), pedregullo (\%) y suelo descubierto (\%), y la cobertura de vegetación (\%) en las dos escalas medidas, además de la pendiente (\%), la orientación relativa al norte (adimensional) y el índice de insolación por intercepción $\left(^{\circ}\right)$. Además, consideramos el término de interacción entre orientación relativa al norte y pendiente por ser un indicador de la insolación asociada a la orientación. Asimismo, consideramos los términos de interacción entre todas las variables y la altitud. No incluimos el efecto principal de la altitud como variable explicativa porque buscamos expresamente individuos con todos los niveles de daño en todas las altitudes.

Utilizamos un procedimiento de selección manual de variables y términos hacia adelante (forward) para encontrar el modelo más parsimonioso (AIC más bajo), y evitamos incluir nuevas variables muy correlacionadas $(r>0.6)$ con aquellas ya incluidas. Buscamos minimizar el AIC independientemente de la significancia de las variables, pero la significancia se consideró al interpretar los resultados. Una vez seleccionado el mejor modelo, repetimos el análisis con todas las variables y términos estandarizados, para conocer el tamaño del efecto.

Finalmente, analizamos la supervivencia tres años después del incendio con modelos lineares generalizados con distribución binomial y enlace logit utilizando las variables asociadas al tamaño y al microambiente como variables independientes, además del efecto principal de la altitud y el nivel de daño (para el caso del molle, consideramos la variable binaria ya descripta). Para la selección de variables y el cálculo del tamaño del efecto seguimos un procedimiento similar al explicado anteriormente. Para todas las pruebas estadísticas utilizamos $\mathrm{R}(\mathrm{R}$ Development Core Team 2017) o Infostat (Di Rienzo et al. 2017) utilizando una interface con R (3.4.0 versión 2017).

\section{RESUltados}

\section{Tamaño y microambiente}

Los molles y espinillos seleccionados tuvieron un rango similar de variación en su tamaño, aunque con una leve tendencia de los espinillos a ser más grandes. Mientras los molles alcanzaron 390 y $350 \mathrm{~cm}$ de altura e índice de volumen, respectivamente, los espinillos llegaron a 450 y $489 \mathrm{~cm}$ para esos mismos parámetros (detalles en Anexo 1). El rango de variación del microambiente también fue similar para ambas especies, desde individuos rodeados casi completamente por roca o suelo desnudo hasta individuos con una cobertura vegetal $\sim 100 \%$ tanto debajo de su copa como en el ambiente circundante. Asimismo, la pendiente y la orientación variaron también de forma similar para ambas especies, desde micrositios casi llanos hasta sitios con pendientes del $30-35 \%$, en todas las orientaciones, mientras que la insolación por intercepción fue menos variable para molle que para espinillo (Anexo 1).

\section{Daño por fuego}

Para el espinillo, el mejor modelo obtenido incluyó la cobertura vegetal en el ambiente circundante, la pendiente, el índice de volumen, la interacción entre la pendiente y la orientación relativa al norte, y la interacción entre la cobertura vegetal y la altitud. La cobertura vegetal tuvo un efecto más fuerte que las otras variables (Tabla 1). Los ejemplares rodeados por más vegetación sufrieron un mayor daño que los ejemplares rodeados por menos vegetación y, por ende, más roca, pedregullo o suelo desnudo. Este efecto de la vegetación fue menos marcado a mayores altitudes que en sectores bajos (Figura 1a). Por otro lado, los espinillos que estaban en laderas orientadas al sur con mucha pendiente sufrieron más daño que aquellos ubicados en sitios llanos o en laderas orientadas norte (Figura 1b). Los resultados

Tabla 1. Modelo linear generalizado (distribución beta y enlace logit) para explicar el nivel de daño por fuego del espinillo (Vachellia caven). Para cada efecto fijo se indica el coeficiente (Coef.), el tamaño del efecto $\left(\mathrm{TE}^{1}\right)$, el valor de z y el $P$.

Table 1. Generalized linear model (beta distribution and logit link) explaining fire damage in espinillo (Vachellia caven). For each fixed effect the coefficient (Coef.), the effect size $\left(\mathrm{TE}^{1}\right)$, and $\mathrm{z}$ and $P$ values are indicated.

\begin{tabular}{|c|c|c|c|c|}
\hline & Coef. & $\mathrm{TE}^{1}$ & $\mathrm{z}$ & $P$ \\
\hline Constante & -0.343 & 0.47 & -0.63 & 0.53 \\
\hline Cobertura vegetal $^{2}$ & 0.042 & 0.71 & 4.07 & $<0.01$ \\
\hline Pendiente & 0.033 & 0.21 & 1.78 & 0.07 \\
\hline Índice de volumen & -0.002 & 0.19 & -2.05 & 0.04 \\
\hline Cobertura vegetal $^{2} \times$ Altitud & $-2.23 \mathrm{E}^{-5}$ & 0.58 & -3.47 & $<0.01$ \\
\hline Pendiente $x$ Orientación N & -0.004 & 0.33 & -3.35 & $<0.01$ \\
\hline
\end{tabular}

$\mathrm{N}=163 ; \mathrm{AIC}=-65.8 ;$ Log-Likelihood=39.91; Deviance=-79.82;

${ }^{1}$ valor absoluto de los coeficientes utilizando las variables y

términos estandarizados; ${ }^{2} \mathrm{en}$ el ambiente circundante (radio de $5 \mathrm{~m}$ alrededor del tronco). 

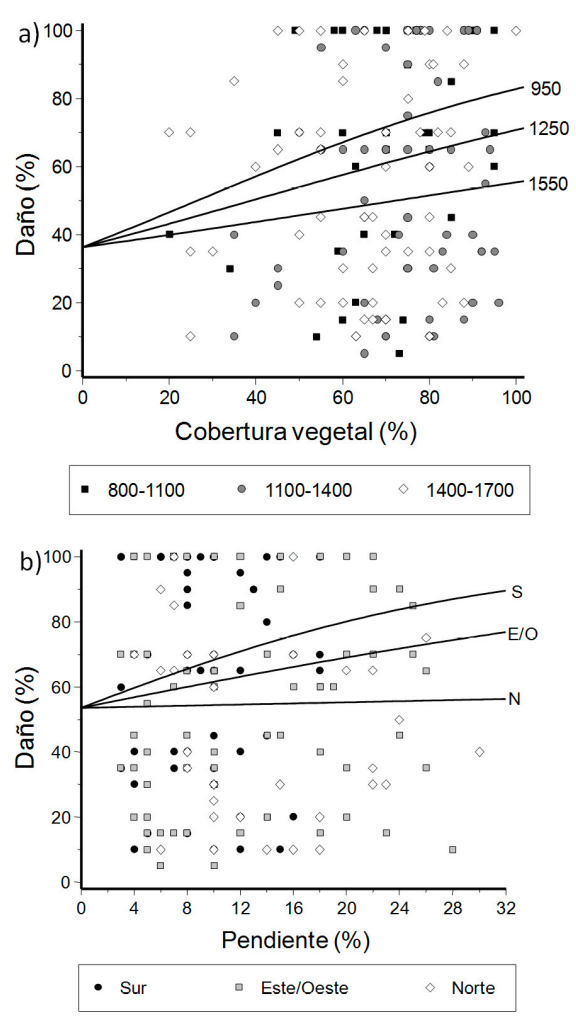

Figura 1. Daño por fuego (\%) en el espinillo (Vachellia caven) en función de cobertura vegetal en el ambiente circundante (a) y la pendiente (b). En (a), los distintos símbolos indican distintos rangos altitudinales (sólo con fines ilustrativos); las tres líneas muestran la relación para el valor central de cada uno de dichos rangos (950, 1250 y 1550 m s. n. m.). En (b), los distintos símbolos indican rangos de orientación relativa al norte (S: entre -1 y -0.34 ; E/O: entre -0.33 y 0.33 ; $\mathrm{N}$ : entre 0.34 y 1 ). Las líneas muestran la relación para el centro de cada rango $(\mathrm{N}=0.7 ; \mathrm{E} / \mathrm{O}=0 ; \mathrm{S}=-0.7)$. En ambos casos, las líneas se obtuvieron a partir de la función logit (multiplicada por 100) aplicada al estimador lineal (EL) indicado en la Tabla 1: Daño(\%)=100/[(1/ $\left.\left.\mathrm{e}^{\wedge \mathrm{EL}}\right)+1\right]$. En (a), la pendiente y la orientación relativa al norte se consideraron como constantes, equivalentes a las medias de todos los datos ( $11 \%$ para la pendiente y -0.12 para la orientación). En (b), la altitud y la cobertura vegetal se consideraron con valores equivalentes a las medias de todos los datos (1250 m s. n. m. y $71 \%$, respectivamente).

Figure 1. Fire damage (\%) in espinillo (Vachellia caven) as a function of plant cover in the surrounding environment (a) and slope (b). In (a), different symbols indicate different ranges established along the altitudinal gradient (only for illustrative purposes); the three lines show the relationship for the central value of each of those ranges $(950,1250$ and $1550 \mathrm{~m}$ a. s. 1). In (b), the different symbols indicate ranges of orientation relative to the north ( $\mathrm{S}$ : between -1 and $-0.34 ; \mathrm{E} / \mathrm{O}$ : between -0.33 and $-0.33 ; \mathrm{N}$ : between 0.34 and 1). The lines show the relationships for the center of each range $(\mathrm{N}=0.7 ; \mathrm{E} / \mathrm{O}=0 ; \mathrm{S}=-0.7)$. In both cases, the lines were obtained from the logit function (multiplied by 100) applied to the linear estimator (EL) indicated in Table1: Fire Damage $(\%)=100 /\left[\left(1 / \mathrm{e}^{\wedge \mathrm{EL}}\right)+1\right]$. In $(\mathrm{a})$, the slope and the orientation relative to the north have been considered as constants, equivalent to the mean of the whole data set ( $11 \%$ for the slope, and -0.12 for the orientation). In (b), altitude and plant cover were considered with values equivalent to the means of the whole data set $(1250 \mathrm{~m}$ a. s. 1 . and $71 \%$, respectively).
Tabla 2. Modelo linear generalizado (distribución binomial y enlace logit) para explicar el nivel de daño (variable binaria, 0: daño parcial, 1: daño total) del molle (Lithraea molleoides). Para cada efecto fijo se indica el coeficiente, el tamaño del efecto $\left(\mathrm{TE}^{1}\right)$, el valor de z y el $P$.

Table 2. Generalized linear model (binomial distribution and logit link) explaining fire damage (binary variable, 0: partial damage, 1: total damage) in molle (Lithraea molleoides). For each fixed effect the coefficients, the effect size $\left(\mathrm{TE}^{1}\right)$, and $\mathrm{z}$ and $P$ values are indicated.

\begin{tabular}{lcccc}
\hline & Coeficiente & $\mathrm{TE}^{1}$ & $\mathrm{z}$ & $P$ \\
\hline Constante & 2.80 & 1.97 & 1.75 & 0.080 \\
Altura & -0.01 & 1.16 & -2.5 & 0.012 \\
Pendiente & 0.20 & 1.29 & 1.5 & 0.134 \\
\hline
\end{tabular}

$\mathrm{N}=48$, AIC=41.47; Log-Likelihood=-17.74;

Deviance $=35.47 ;{ }^{1}$ valor absoluto de los coeficientes

utilizando las variables y términos estandarizados.

también mostraron que los individuos más grandes sufrieron menos daño, pero el efecto del tamaño fue débil en comparación con los otros efectos. Para el molle, el mejor modelo mostró que los árboles más altos sufrieron menos daño. Además, aquellos ubicados en sitios con más pendiente tendieron a dañarse más, aunque este efecto no fue significativo (Tabla 2, Figura 2).

\section{Supervivencia}

Después de tres años de ocurrido el incendio, el $94 \%$ de los espinillos seleccionados sobrevivieron (sólo 9 murieron, Figura 3). El modelo más parsimonioso para explicar la supervivencia incluyó la altura del ejemplar y la proporción de roca en el ambiente circundante (Tabla 3). Estos resultados indican que los árboles más altos sobrevivieron más, y que estar rodeados por roca también tendió a favorecerlos, aunque esta tendencia no fue significativa. En el caso del molle, el $92 \%$ de los ejemplares seleccionados sobrevivieron hasta tres años después del incendio (murieron 4

Tabla 3. Modelo linear generalizado (distribución binomial y enlace logit) para explicar la supervivencia del espinillo (Vachellia caven). Para cada efecto fijo se indica el coeficiente, el tamaño del efecto $\left(\mathrm{TE}^{1}\right)$, el valor de z y el $P$.

Table 3. Generalized linear model (binomial distribution and logit link) explanatory of the survival in espinillo (Vachellia caven). For each fixed effect the coefficients, the effect size $\left(\mathrm{TE}^{1}\right)$, and $\mathrm{z}$ and $P$ values are indicated.

\begin{tabular}{lcccc}
\hline & Coeficiente & $\mathrm{TE}^{1}$ & $\mathrm{z}$ & $P$ \\
\hline Constante & -0.10 & 3.18 & -0.08 & 0.94 \\
Altura & 0.01 & 0.80 & 1.96 & 0.05 \\
Roca $^{2}$ & 0.04 & 0.59 & 1.28 & 0.20 \\
\hline
\end{tabular}

$\mathrm{N}=163$; AIC=69.84; Log-Likelihood=-31.92;

Deviance $=63.84 ;{ }^{1}$ valor absoluto de los coeficientes utilizando las variables y términos estandarizados; ${ }^{2} \mathrm{en}$ el ambiente circundante (radio de $5 \mathrm{~m}$ alrededor del tronco). 


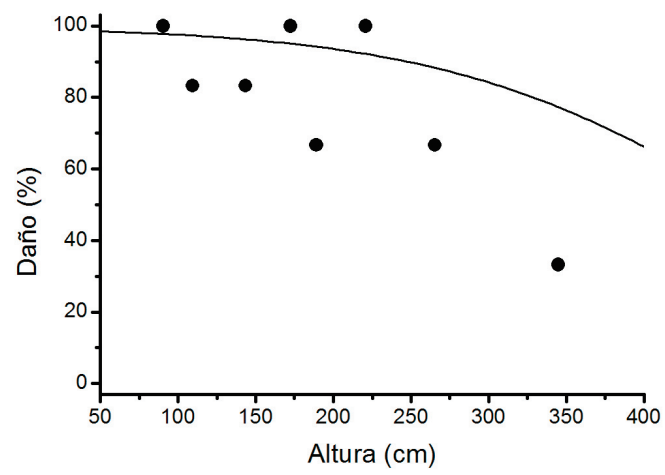

Figura 2. Daño en el molle (Lithraea molleoides) en función de la altura del ejemplar. Los ejemplares se agruparon en categorías según su altura (menos de $100 \mathrm{~cm}$ y de ahí en más en categorías cada $50 \mathrm{~cm}$, sólo con fines ilustrativos). Cada punto representa el promedio de altura de cada categoría y la proporción (\%) de árboles dentro de dicha categoría que sufrió un daño total. La línea fue obtenida a partir de la función logit (multiplicada por 100) aplicada al estimador lineal (EL) indicado en la Tabla 2: Daño $(\%)=100 /\left[\left(1 / \mathrm{e}^{\mathrm{EL}}\right)+1\right]$. La pendiente se consideró como una constante equivalente a la media de todos los datos $(9.37 \%)$

Figure 2. Fire damage in molle (Lithraea molleoides) as a function of tree height. The individuals have been ranked in classes according to their height (less than $100 \mathrm{~cm}$ and from there on in classes every $50 \mathrm{~cm}$, only for illustrative purposes). Each point represents the average height of each class, and the proportion (\%) of trees within each class that suffered a total damage. The line was obtained from the logit function (multiplied by 100) applied to the linear estimator (EL) indicated in Table 2: Fire Damage $(\%)=100 /\left[\left(1 / \mathrm{e}^{\mathrm{EL}}\right)+1\right]$. The slope was considered as a constant equivalent to the mean of all the data set $(9.37 \%)$.

individuos). El modelo más parsimonioso para explicar la supervivencia incluyó el nivel de daño (variable binaria) y la proporción de roca en el ambiente circundante (Tabla 4). Los individuos rodeados por más roca y que fueron sólo parcialmente dañados por el fuego mostraron una tendencia no significativa a sobrevivir más.

Tabla 4. Modelo lineal generalizado (distribución binomial y enlace logit) para explicar la supervivencia del molle (Lithraea molleoides). Para cada efecto fijo se indica el coeficiente, el tamaño del efecto $\left(\mathrm{TE}^{1}\right)$, el valor de z y el $P$.

Table 4. Generalized linear model (binomial distribution and logit link) explaining survival in molle (Lithraea molleoides). For each fixed effect the coefficients, the effect size $\left(\mathrm{TE}^{1}\right)$, and $\mathrm{z}$ and $P$ values are indicated.

\begin{tabular}{lcccc}
\hline & Coeficiente & $\mathrm{TE}^{1}$ & $\mathrm{z}$ & $P$ \\
\hline Constante & 19.2 & 6.06 & 0.01 & 0.99 \\
Daño $^{2}$ & -17.8 & 7.32 & -0.01 & 0.99 \\
Roca $^{3}$ & 0.04 & 0.97 & 1.34 & 0.18
\end{tabular}

$\mathrm{N}=48 ; \mathrm{AIC}=29.19$; Log-Likelihood=-11.60;

Deviance=23.19; ${ }^{1}$ valor absoluto de los coeficientes utilizando las variables y términos estandarizados; ${ }^{2}$ variable binaria (0: daño parcial, 1: daño total); ${ }^{3} \mathrm{en}$ el ambiente circundante (radio de $5 \mathrm{~m}$ alrededor del tronco).

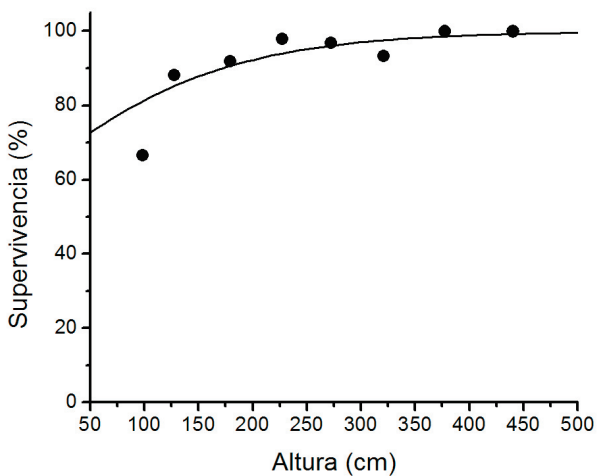

Figura 3. Supervivencia (\%) del espinillo después de tres años del incendio en función de la altura del ejemplar. Los árboles se agruparon en categorías según su altura (menor o igual a $100 \mathrm{~cm}$ y de ahí en más en categorías cada $50 \mathrm{~cm}$, sólo con fines ilustrativos). Cada punto representa el promedio de altura de cada categoría y la proporción (\%) de árboles dentro de dicha categoría que sobrevivió al incendio. La línea se obtuvo a partir de la función logit (multiplicada por 100) aplicada al estimador lineal (EL) indicado en la Tabla 3: Supervivencia $(\%)=100 /\left[\left(1 / \mathrm{e}^{\mathrm{EL}}\right)+1\right]$. La roca alrededor del individuo se consideró como una constante equivalente a la media de todos los árboles $(14.35 \%)$.

Figure 3. Survival (\%) of espinillo three years after the fire as a function of tree height. The individuals have been grouped in classes according to their height (equal or less than $100 \mathrm{~cm}$ and from there on in classes each 50 $\mathrm{cm}$, only for illustrative purposes). Each point represents the average height of each class, and the proportion (\%) of trees within each class that survived after the fire. The line was obtained from the logit function (multiplied by 100) applied to the linear estimator (EL) indicated in Table 3: Survival $(\%)=100 /\left[\left(1 / \mathrm{e}^{\mathrm{EL}}\right)+1\right]$. The rock cover surrounding the trees was considered as a constant equivalent to the mean of all the individuals (14.35\%).

\section{DisCUSIÓN}

Nuestro estudio mostró que a pesar del daño total o parcial que produjo el fuego en los tejidos de los árboles, la supervivencia de los individuos seleccionados de ambas especies fue muy alta, en coincidencia con otros estudios en la región. Esto pone en evidencia la resiliencia elevada del sistema ante este disturbio (Torres et al. 2014; Herrero et al. 2015; Lipoma et al. 2016). El escape parcial de algunos ejemplares también ponedemanifiesto cierta resistencia de las comunidades leñosas al fuego. El tamaño de los individuos y las características del microambiente afectaron el daño y la posterior supervivencia, lo que indica, entonces, que estos factores influyen sobre la estabilidad del sistema ante el disturbio por fuego.

\section{Daño por fuego}

Tal como esperábamos, los molles y los espinillos más pequeños sufrieron más daño que los más grandes, en coincidencia 
con otros estudios donde se observaron patrones similares (Cable 1965; Fernandes et al. 2008; Brando et al. 2012). La altura fue más importante para molle, mientras que el volumen fue más importante para espinillo. Las diferencias arquitecturales y en inflamabilidad de los tejidos entre ambas especies podrían explicar estos patrones (Blackhall et al. 2012; Bianchi and Defossé 2015). Al igual que otros arbustos o árboles pequeños, el espinillo tiene tejidos muy inflamables en comparación con otras leñosas, y una estructura más arbustiva y ramificada desde la base (Jaureguiberry et al. 2011; Blackhall et al. 2012). Esto podría facilitar que el fuego alcance las ramas bajas y se propague hacia arriba por la copa sin limitaciones una vez que empieza la combustión. Sin embargo, como se trata de un árbol semideciduo y con una copa abierta, la propagación lateral del fuego entre ramas diferentes podría verse dificultada, lo que explicaría por qué los espinillos más voluminosos sufrieron proporcionalmente menos daño. En el caso del molle, que tiende a estar menos ramificado desde la base y tiene una estructura de la copa más compacta, es posible que haya más limitaciones a la propagación vertical del fuego que a la propagación lateral, lo cual permite que algunos de los árboles más altos escapen parcialmente.

Como confirmación de nuestras predicciones, los espinillos rodeados por menos vegetación pudieron escapar más del fuego, mientras que para el molle esta variable no resultó seleccionada. En general, el espinillo deja pasar más luz y tiene más gramíneas debajo de la copa y en sus alrededores que el molle (Giorgis et al. 2017). Dado que las gramíneas tienen una alta inflamabilidad (Jaureguiberry et al. 2011; Bianchi et al. 2014), cuando su cobertura es alta pueden producir mucho daño en el ejemplar leñoso. En el caso del molle, es probable que al tener en general una cobertura menos inflamable debajo de su copa y en sus alrededores, esta variable haya resultado menos importante y no fue detectada. En coincidencia con los patrones hallados aquí para espinillo, en otros estudios se encontró que, en sitios protegidos por roca y, por ende, con menos vegetación, los árboles escapan más del fuego ya que las llamas se mantienen más alejadas de sus tejidos (Renison et al. 2002; Alinari et al. 2015; Landesmann et al. 2015). Por estas razones, es de esperar que en ambientes rocosos la cobertura de plantas leñosas sea mayor que en ambientes menos rocosos, un patrón ya observado tanto en las Sierras de
Córdoba como en otros lugares. En las Sierras deCórdoba, los bosques de tabaquillo(Polylepis australis) tienen una distribución restringida a lugares protegidos del fuego o de la herbivoría, como son los valles o sitios rocosos (Renison et al. 2002, 2006; Cingolani et al. 2008; Alinari et al. 2015). En el sur de Brasil se observó una expansión de árboles del género Araucaria sobre los pastos en los micrositios menos afectados por el fuego y el ganado (Carlucci et al. 2011). Algo similar ocurre en el sur de la Argentina, donde se observa la presencia de remanentes de Austrocedrus chilensis en lugares donde las condiciones ambientales protegen a los individuos del fuego (e.g., en ambientes rocosos y zonas más escarpadas) (Landesmann et al. 2015).

Una menor pendiente disminuyó el daño en ambas especies; en el caso del molle, la relación no llegó a ser significativa, y en el caso del espinillo, esta relación dependió de la orientación de la ladera. Esto podría explicarse por la mayor humedad del suelo (y, por ende, de los combustibles) en los sitios más llanos, tal como habíamos postulado (Bianchi and Defossé 2015). Sin embargo, los resultados obtenidos para las variables relacionadas con la insolación no apoyan esta interpretación. La insolación por intercepción no influyó en el daño por fuego de ninguna de las dos especies, mientras que la orientación de la ladera tampoco tuvo un efecto sobre molle, y el efecto sobre espinillo fue opuesto al esperado: los individuos ubicados en laderas norte sufrieron menos daño que los ubicados en laderas sur. Es posible que los resultados encontrados se relacionen con la reducción en la altura de la vegetación producida por el ganado (Briggs et al. 2002; Blackhall et al. 2015b). Si bien están presentes en todas partes, los herbívoros domésticos suelen seleccionar los sitios más llanos, tanto en el área de estudio como en otros lugares (von Müller et al. 2017 y trabajos citados ahí). En el área de estudio, además de consumir la vegetación y reducir su tamaño, promueven el reemplazo de pastos altos por dicotiledóneas y gramíneas bajas, en particular a mayores altitudes y en laderas norte (Cingolani et al. 2008, 2014). Por este motivo es posible que los ejemplares de espinillo ubicados en laderas norte y a mayor altitud, incluso teniendo alta cobertura vegetal debajo de su copa, hayan estado más protegidos del fuego. En otros sistemas se encontró que la presencia de ganado disminuye la incidencia y severidad de los fuegos al eliminar biomasa y reducir la altura de la vegetación (e.g., Briggs et al. 2002). 


\section{Supervivencia}

La supervivencia de los individuos mayores a $80 \mathrm{~cm}$ seleccionados fue alta para ambas especies, y no pudimos detectar una relación con el nivel de daño. En cambio, detectamos que la mayor altura pre-fuego facilitó la supervivencia en el espinillo. Es posible que los árboles más grandes tengan más reservas subterráneas y, por lo tanto, más probabilidad de recuperarse. En especies de bosque serrano, incluyendo al espinillo, se observó que el mayor tamaño del ejemplar favorece al vigor del rebrote (Gurvich et al. 2005; Herrero et al. 2015). Los rebrotes poco vigorosos podrían ser más susceptibles a la sequía, a las heladas y a la herbivoría, y producir la muerte del individuo dentro de los primeros tres años después del incendio (Torres et al. 2014). En estudios realizados en otros ecosistemas también se detectó que los árboles más grandes dentro de una misma especie tienen mayores probabilidades de sobrevivir (Fernandes et al. 2008; Brando et al. 2012). Si bien la supervivencia de los árboles mayores a $80 \mathrm{~cm}$ fue muy alta, es posible que la supervivencia de los árboles más pequeños, no incluidos en el estudio, haya sido más baja. Esto es esperable al menos para el espinillo, que mostró una relación significativa entre el tamaño y la supervivencia.

\section{Consideraciones finales}

Si bien el fuego no suele causar la muerte de los individuos leñosos en las Sierras de Córdoba, cuando los tejidos resultan muy dañados se reduce considerablemente la biomasa leñosa y los árboles rebrotan desde la base del tallo o desde las raíces (Renison et al. 2002; Torres et al. 2014; Alinari et al. 2015; Lipoma et al. 2016). De este modo, los bosques retornan a un estado sucesional temprano con árboles pequeños y alta abundancia de gramíneas y dicotiledóneas herbáceas que, a su vez, favorecen la propagación de los siguientes fuegos (Giorgis et al. 2013, 2017; Lipoma et al. 2016; Argañaraz et al. 2015a). A través del presente estudio pudimos describir algunos de los mecanismos asociados a esta retroalimentación positiva entre el fuego y la vegetación (Tepley et al. 2018). Observamos que el daño sobre las especies leñosas es mayor cuando son pequeñas y están rodeadas por una alta cobertura de especies herbáceas, una situación habitual pocos años después de haber ocurrido un incendio (Giorgis et al. 2013). Por lo tanto, si se produce una nueva ignición en esta etapa, la vegetación herbácea favorece la propagación del fuego y el daño sobre las especies leñosas es máximo. A medida que transcurre el tiempo, si se logra prevenir un nuevo fuego, la sucesión avanza, los espinillos y los molles aumentan de tamaño y la cobertura herbácea disminuye. De este modo se reduce paulatinamente el daño y la probabilidad de muerte si ocurre un incendio. Si el proceso continúa, los molles finalmente se hacen dominantes y los espinillos, así como las gramíneas, disminuyen o desaparecen por la falta de luz (Giorgis et al. 2013, 2017). De este modo, los bosques bien desarrollados tienen una mayor resistencia al fuego que los arbustales y los pastizales (Argañaraz et al. 2015a).

Al igual que en otros casos, en nuestro sistema, una vez que se conforma un bosque maduro es difícil que se queme (Tepley et al. 2018). Sin embargo, la suma de años muy secos con el incremento de las igniciones por causas antropogénicas puede desencadenar un fuego de alta severidad que queme por completo la biomasa aérea del bosque y promueva un reemplazo por comunidades sucesionales tempranas (Tepley et al. 2018; Argibay and Renison 2018). En otros casos, este cambio se puede producir de forma paulatina cuando otros disturbios antropogénicos (e.g., el ganado o la tala selectiva) modifican la estructura del bosque y las características del sotobosque, promoviendo una inflamabilidad mayor (Uhl and Kauffman 1990; Raffaele et al. 2011; Blackhall et al. 2015b). Esto puede producir una apertura parcial del dosel, lo cual, a su vez, facilita la entrada de nuevos fuegos. Esto genera el mecanismo de retroalimentación positiva que finalmente desemboca en el reemplazo completo del bosque por matorrales o pastizales que se mantienen indefinidamente porque no se logran evitar los fuegos durante el tiempo suficiente para que el bosque madure (Uhl and Kauffman 1990; Blanco et al. 2014; Kitzberger et al. 2016).

Agradecimientos. Agradecemos a las familias Dorado y López del Prato por permitirnos estudiar los incendios en sus propiedades, y por sus cálidos recibimientos luego de cada jornada de trabajo. También agradecemos a Thomas Kitzberger y Alicia Sércic por sus ideas y continuos aportes durante los años en que duró la Tesis que dio origen a este trabajo, a Guillermo Defossé por brindar sus conocimientos sobre la dinámica de fuegos y a Iván Barberá por su ayuda con el R. Este trabajo fue realizado gracias a una beca de CONICET 
a la primera autora y al financiamiento del Inter-American Institute for Global Change Research, CONICET (PIP 112-201201-00164),
Ministerio de Ciencia y Tecnología de la provincia de Córdoba y proyecto binacional DFG (Alemania).

\section{REFERENCIAS}

Alinari, J. 2017. ¿Puede el fuego limitar la distribución altitudinal de las principales especies del Bosque Serrano de la Provincia de Córdoba? Universidad Nacional de Córdoba, Córdoba, Argentina.

Alinari, J., A. R. von Müller, and D. Renison. 2015. The contribution of fire damage to restricting high mountain Polylepis australis forests to ravines: Insights from an un-replicated comparison. Ecología Austral 25:11-18.

Argañaraz, J. P., G. Gavier Pizarro, M. Zak, and L. M. Bellis. 2015a. Fire regime, climate, and vegetation in the Sierras de Cordoba, Argentina. Fire Ecology 11:55-73. https://doi.org/10.4996/fireecology.1101055.

Argañaraz, J. P., G. Gavier Pizarro, M. Zak, M. A. Landi, and L. M. Bellis. 2015b. Human and biophysical drivers of fires in Semiarid Chaco mountains of Central Argentina. Science of the Total Environment 520:1-12. https://doi.org/ 10.1016/j.scitotenv.2015.02.081.

Argibay, D. S., and D. Renison. 2018. Efecto del fuego y la ganadería en bosques de Polylepis australis (Rosaceae) a lo largo de un gradiente altitudinal en las montañas del centro de la Argentina. Bosque 39(1):145-150. https: / / doi.org/ 10.4067/S0717-92002018000100145.

Bianchi, L. O., G. E. Defossé, M. Dentoni, C. Kunst, R. Ledesma, and S. Bravo. 2014. Dinámica de la humedad de los combustibles y su relación con la ecología y el manejo de fuego en la región chaqueña occidental (Argentina) I: conceptos básicos. Revista de Investigaciones Agropecuarias 40(2):154-164.

Bianchi, L. O, and G. E. Defossé. 2015. Live fuel moisture content and leaf ignition of forest species in Andean Patagonia, Argentina. International Journal of Wildland Fire 24:340-348. https:/ / doi.org/10.1071/WF13099.

Blackhall, M., E. Raffaele, and T. T. Veblen. 2012. Is foliar flammability of woody species related to time since fire and herbivory in northwest Patagonia, Argentina? Journal of Vegetation Science 23:931-941. https://doi.org/10.1111/ j.1654-1103.2012.01405.x.

Blackhall, M., E. Raffaele, and T. T. Veblen. 2015a. Efectos combinados del fuego y el ganado en matorrales y bosques del noroeste patagónico. Ecología Austral 25:1-10.

Blackhall, M., T. T. Veblen, and E. Raffaele. 2015b. Recent fire and cattle herbivory enhance plant-level fuel flammability in shrublands Journal of Vegetation Science 26:123-133. https://doi.org/10.1111/jvs.12216.

Blanco, C. C., S. Scheiter, E. Sosinski, A. Fidelis, M. Anand, and V. D. Pillar. 2014. Feedbacks between vegetation and disturbance processes promote long-term persistence of forest-grassland mosaics in south Brazil. Ecological Modelling 291:224-232. https://doi.org/10.1016/j.ecolmodel.2014.07.024.

Bond, W. J., F. I. Woodward, and G. F. Midgley. 2005. The global distribution of ecosystems in a world without fire. New Phytologist 165:525-538. https://doi.org/10.1111/j.1469-8137.2004.01252.x.

Brando, P. M., D. C. Nepstad, J. K. Balch, B. Bolker, M. C. Christman, M. Coe, and F. E. Putz. 2012. Firelinduced tree mortality in a Neotropical forest: the roles of bark traits, tree size, wood density and fire behavior. Global Change Biology 18:630-641. https:/ / doi.org/10.1111/j.1365-2486.2011.02533.x.

Briggs, J. M., A. K. Knapp, and B. L. Brock. 2002. Expansion of woody plants in tallgrass prairie: a fifteen-year study of fire and fire-grazing Interactions. The American Midland Naturalist 147(2):287-294. https:/ / doi.org/10.1674/00030031(2002)147[0287:EOWPIT]2.0.CO;2.

Cabido, M., G. Funes, E. Pucheta, F. Vendranimi, and S. Díaz. 1998. A chorological analysis of the mountains from Central Argentina. Is all what we call Sierra Chaco really Chaco? Contribution to the study of the flora and vegetation of the Chaco. XII. Candollea 53:321-331.

Cabido, M., S. R. Zeballos, M. Zak, M. L. Carranza, M. A. Giorgis, J. J. Cantero, and A. T. R. Acosta. 2018. Native woody vegetation in central Argentina: classification of Chaco and Espinal forests. Applied Vegetation Science 21:298-311. https://doi.org/10.1111/avsc.12369.

Cable, W. R. 1965. Damage to mesquite, lehmann lovegrass, and black grama by a hot June fire. Journal of Range Management 18:326-329. https://doi.org/10.2307/3895424.

Capó, E. A., R. Aguilar, and D. Renison. 2016. Livestock reduces juvenile tree growth of alien invasive species with a minimal effect on natives: a field experiment using exclosures. Biological Invasions 18:2943-2950. https:/ /doi.org/ 10.1007/s10530-016-1185-3.

Carlucci, M. B., L. da S. Duarte, and V. D. Pillar. 2011. Nurse rocks influence forest expansion over native grassland in southern Brazil. Journal of Vegetation Science 22:111-119. https://doi.org/10.1111/j.1654-1103.2010.01229.x.

Cingolani, A. M., D. Renison, P. A. Tecco, D. E. Gurvich, and M. Cabido. 2008. Predicting cover types in a mountain range with long evolutionary grazing history: a GIS approach. Journal of Biogeography 35:538-551. https:/ / doi.org/ 10.1111/j.1365-2699.2007.01807.x.

Cingolani, A. M., M. V. Vaieretti, M. A. Giorgis, M. Poca, P. A. Tecco, and D. E. Gurvich. 2014. Can livestock grazing maintain landscape diversity and stability in an ecosystem that evolved with wild herbivores? Perspectives in Plant Ecology, Evolution and Systematics 16:143-153. https:/ / doi.org/10.1016/j.ppees.2014.04.002.

Colladon, L., and I. Pazos. 2014. Anuario pluviométrico 1992-2012. Cuenca del río San Antonio, Sistema del Río Suquía, Provincia de Córdoba. Instituto Nacional del Agua y del Ambiente (INA) y Centro de Investigaciones de la Región Semiárida (CIRSA). Córdoba, Argentina.

Di Rienzo, J. A., F. Casanoves, M. G. Balzarini, L. González, M. Tablada, and C. W. Robledo. 2017. InfoStat. Córdoba, 
Argentina. Grupo InfoStat.

Fernandes, P. M., J. A. Vega, E. Jiménez, and E. Rigolot. 2008. Fire resistance of European pines. Forest Ecology and Management 256:246-255. https://doi.org/10.1016/j.foreco.2008.04.032.

Flatley, W. T., C. W. Lafon, and H. D. Grissino-Mayer.2011. Climatic and topographic controls on patterns of fire in the southern and central Appalachian Mountains, USA. Landscape Ecology 26:195-209. https://doi.org/10.1007/ s10980-010-9553-3.

Giorgis, M. A., A. M. Cingolani, and M. Cabido. 2013. El efecto del fuego y las características topográficas sobre la vegetación y las propiedades del suelo en la zona de transición entre bosques y pastizales de las sierras de Córdoba, Argentina. Boletín de la Sociedad Argentina de Botánica 48:493-513.

Giorgis, M. A., A. M. Cingolani, D. E. Gurvich, P. A. Tecco, J. Chiapella, F. Chiarini, and M. Cabido. 2017. Changes in floristic composition and physiognomy are decoupled along elevation gradients in central Argentina. Applied Vegetation Science 20:558-571. https://doi.org/10.1111/avsc.12324.

Gurvich, D. E., L. Enrico, and A. M. Cingolani. 2005. Linking plant functional traits with post-fire sprouting vigour in woody species of central Argentina. Austral Ecology 30:789-796. https://doi.org/10.1111/j.1442-9993.2005.01522.x. https: //doi.org/10.1111/j.1442-9993.2005.01529.x.

Herrero, M. L., R. C. Torres, and D. Renison. 2015. Do Wildfires Promote Woody Species Invasion in a Fire-Adapted Ecosystem? Post-fire Resprouting of Native and Non-native Woody Plants in Central Argentina. Environmental Management 57:308-317. https://doi.org/10.1007/s00267-015-0616-8.

Jaureguiberry, P., G. Bertone, and S. Díaz. 2011. Device for the standard measurement of shoot flammability in the field. Austral Ecology 36:821-829. https://doi.org/10.1111/j.1442-9993.2010.02222.x.

Keeley, J. E. 2009. Fire intensity, fire severity and burn severity: a brief review and suggested usage. International Journal of Wildland Fire 18:116-126. https://doi.org/10.1071/WF07049.

Kitzberger, T., G. L. W. Perry, J. Paritsis, J. H. Gowda, A. J. Tepley, A. Holz, and T. T. Veblen. 2016. Fire-vegetation feedbacks and alternative states: common mechanisms of temperate forest vulnerability to fire in southern South America and New Zealand, New Zealand Journal of Botany 54:247-272. https://doi.org/10.1080/0028825X.2016.1151903.

Kupferschmid, A. D., and H. Bugmann. 2005. Effect of microsites, logs and ungulate browsing on Picea abies regeneration in a mountain forest. Forest Ecology and Management 205:251-265. https://doi.org/10.1016/j.foreco.2004.10.008.

Kurtz, F. 1904. Flora de Córdoba. Pp. 270-343 en M. E. Río and L. Achával (eds.). Geografía de la Provincia de Córdoba. Volumen 1. Compañía Sudamericana de Billetes de Banco, Buenos Aires.

Landesmann, J. B., J. H. Gowda, L. A. Garibaldi, and T. Kitzberger. 2015. Survival, growth and vulnerability to drought in fire refuges: implications for the persistence of a fire-sensitive conifer in northern Patagonia. Oecologia 179:11111122. https://doi.org/10.1007/s00442-015-3431-2.

Lipoma, M. L., D. E. Gurvich, C. Urcelay, and S. Díaz. 2016. Plant community resilience in the face of fire: experimental evidence from a semi-arid shrubland. Austral Ecology 41:501-511. https://doi.org/10.1111/aec.12336.

Marañón, T., J. J. Camarero, J. Castro, M. Díaz, J. M. Espelta, A. Hampe, P. Jordano, F. Valladares, M. Verdú, and R. Zamora. 2004. Heterogeneidad ambiental y nicho de regeneración. Pp. 69-99 en Ecología del bosque mediterráneo en un mundo cambiante. Ministerio de Medio Ambiente, Madrid.

Marcora, P. I., I. Hensen, D. Renison, P. Seltmann, and K. Wesche. 2008. The performance of Polylepis australis trees along their entire altitudinal range: implications of climate change for their conservation. Diversity and Distributions 14:630-636. https://doi.org/10.1111/j.1472-4642.2007.00455.x.

Martínez, G. A., M. D. Arana, A. J. Oggero, and E. S. Natale. 2017. Biogeographical relationships and new regionalisation of high-altitude grasslands and woodlands of the central Pampean Ranges (Argentina), based on vascular plants and vertebrates. Australian Systematic Botany 29:473-488. https://doi.org/10.1071/SB16046.

Oliveira, J. M., and V. D. Pillar. 2004. Vegetation dynamics on mosaics of Camposand Araucaria forest between 1974 and 1999 in Southern Brazil. Community Ecology 5:197-202. https://doi.org/10.1556/ComEc.5.2004.2.8.

Ordoñez, J. L., J. Retana, and J. M. Espelta. 2005. Effects of tree size, crown damage, and tree location on post-fire survival and cone production of Pinus nigra trees. Forest Ecology and Management 206:109-117. https://doi.org/ 10.1016/j.foreco.2004.10.067.

Parker, A. J. 1982. The topographic relative moisture index: an approach to soil-moisture assessment in mountain terrain. Physical Geography 3(2):160-168. https:// doi.org/10.1080/02723646.1982.10642224.

R Development Core Team. 2017. A language and environment for statistical computing. R Foundation for Statistical Computing, Vienna, Austria. URL: www.R-project. org.

Raffaele, E., T. T. Veblen, M. Blackhall, and N. Tercero-Bucardo. 2011. Synergistic influences of introduced herbivores and fire on vegetation change in northern Patagonia, Argentina. Journal of Vegetation Science 22:59-71. https:// doi.org/10.1111/j.1654-1103.2010.01233.x.

Renison, D., A. M. Cingolani, and R. Suárez. 2002. Efectos del fuego sobre un bosquecillo de Polylepis australis (Rosaceae) en las montañas de Córdoba, Argentina. Revista Chilena de Historia Natural 75:719-727. https://doi.org/10.4067/ S0716-078X2002000400007.

Renison, D., I. Hensen, R. Suárez, and A. M. Cingolani. 2006. Cover of Polylepis woodlands and shrublands in the mountains of central Argentina: human or environmental influence? Journal of Biogeography 33:876-887. https: //doi.org/10.1111/j.1365-2699.2006.01455.x.

Tecco, P. A., A. I. Pais-Bosch, G. Funes, P. I. Marcora, S. R. Zeballos, M. Cabido, and C. Urcelay. 2015. Mountain invasions on the way: are there climatic constraints for the expansion of alien woody species along an elevation gradient in 
Argentina? Journal of Plant Ecology 9:380-392. https://doi.org/10.1093/jpe/rtv064.

Tepley, A. J., E. Thomann, T. T. Veblen, G. L. W. Perry, A. Holz, J. Paritsis, T. Kitzberger, and K. J. Anderson-Teixeira. 2018. Influences of fire-vegetation feedbacks and post-fire recovery rates on forest landscape vulnerability to altered fire regimes. Journal of Ecology 106:1925-1940. https:/ / doi.org/10.1111/1365-2745.12950.

Torres, R. C., M. A. Giorgis, C. Trillo, L. Volkmann, P. Demaio, J. Heredia, and D. Renison. 2014. Postlfire recovery occurs overwhelmingly by resprouting in the Chaco Serrano forest of Central Argentina. Austral Ecology 39:346-354.

Torres, R. C., M. A. Giorgis, C. Trillo, L. Volkmann, P. Demaio, J. Heredia, and D. Renison. 2015. Supervivencia y crecimiento de especies con distinta estrategia de vida en plantaciones de áreas quemadas y no quemadas: un estudio de caso con dos especies leñosas en el Chaco Serrano, Argentina. Ecología Austral 25:135-143. https://doi.org/ $10.1111 /$ aec.12084.

Türkmen, N., and A. Düzenli. 2005. Changes in floristic composition of Quercus coccifera macchia after fire in the Çukurova region (Turkey). Annales Botanici Fennici 42:453-460.

Uhl, C., and J. B. Kauffman. 1990. Deforestation, Fire Susceptibility, and Potential Tree Responses to Fire in the Eastern Amazon. Ecology 71:437-449. https://doi.org/10.2307/1940299.

USDA. 2017. Soil Science Division Staff. Soil survey manual. C. Ditzler, K. Scheffe and H. C. Monger (eds.). USDA Handbook 18. Government Printing Office, Washington, DC.

Veblen, T. T., T. Kitzberger, R. Villalba, and J. Donnegan. 1999. Fire history in northern Patagonia: the roles of humans and climatic variation. Ecological Monographs 69:47-67. https:/ / doi.org/10.1890/0012-9615(1999)069[0047: FHINPT]2.0.CO;2.

von Müller, A. R., D. Renison, and A. M. Cingolani. 2017. Cattle landscape selectivity is influenced by ecological and management factors in a heterogeneous mountain rangeland. Rangeland Journal 39:1-14. https://doi.org/10.1071/ RJ15114. 